\title{
Improvement on a 2 × 2 Elements High-Gain Circularly Polarized Antenna Array
}

\author{
C. Liu, A. Yan, C. Yu, and T. Xu \\ School of Electronics and Information Engineering, Sichuan University, Chengdu 610064, China \\ Correspondence should be addressed to C. Liu; cjliu@scu.edu.cn
}

Received 27 January 2015; Revised 12 May 2015; Accepted 30 May 2015

Academic Editor: Angelo Liseno

Copyright (C) 2015 C. Liu et al. This is an open access article distributed under the Creative Commons Attribution License, which permits unrestricted use, distribution, and reproduction in any medium, provided the original work is properly cited.

\begin{abstract}
A novel antipodal Vivaldi antenna with tapering serrated structure at the edges is proposed. Compared with traditional Vivaldi antennas without serrated structure, the gain of the designed antenna is significantly improved in the desired frequency band (4.5$7.5 \mathrm{GHz}$ ). In addition, a $2 \times 2$ Vivaldi antenna array with an orthorhombic structure is designed and fabricated to achieve a circular polarization $(\mathrm{CP})$ characteristic. With this configuration, the $3 \mathrm{~dB}$ axial ratio bandwidth of the array reaches about $42 \%$ with respect to the center frequency of $6 \mathrm{GHz}$ and a high gain is achieved as well. The novel Vivaldi antenna and CP antenna array both have ultrawide band (UWB) and high-gain characteristics, which may be applied to the field of commercial communication, remote sensing, and so forth.
\end{abstract}

\section{Introduction}

The rapid development of wireless communication systems, including commercial or military communication, remote sensing, and various point-to-point links, has brought in the demand of ultrawide band (UWB) $[1,2]$ and high-gain antennas. As an essential part of a communication system, many kinds of antennas have been extensively studied since the Federal Communications Commission (FCC) allocated the 3.1-10.6 GHz band to commercial purposes in 2002 .

Among existing high-gain UWB antennas, Vivaldi antennas possess some attractive properties including broad bandwidth, compactness, and highly directive patterns. Compared with some other UWB antennas, such as a ridge horn antenna, a Vivaldi antenna can be simply fabricated without a UWB balun. Vivaldi antenna has been paid great attentions to since its first appearance in 1979 [3] and is widely used in wireless and radar applications [4]. Many improvements on Vivaldi antenna have been made in the last three decades to achieve some desired characteristics. A better radiation pattern is formed by introducing corrugated edges $[5,6]$; the shape of the corrugated edges is very critical for design [7]. This structure alters the phases of currents flowing along the outer part of the antenna substrate and changes the electric field at the edges of this substrate $[8,9]$. Additionally, low cross polarization and highly directive patterns can be got through modifying the traditional Vivaldi antenna by exponentially tapering inner and outer edges [10].

In $[11,12]$, the gain or the band width is still limited. Thus, we designed a novel tapering periodic serrated structure in this paper in which a function of gradient tapering serrated structures was used for design. The designed antenna promotes the gain from $9 \mathrm{dBi}$ to $11.5 \mathrm{dBi}$ in the desired frequency band $(4.5-7.5 \mathrm{GHz})$. Unlike previous papers, we focus on combining a $6 \mathrm{~dB}$ Wilkinson power divider through $2 \times 2$ array with orthorhombic structure to achieve a high-gain broadband directional circularly polarized antenna array. At last the $3 \mathrm{~dB}$ axial ratio bandwidth is up to $42 \%$ with respect to the center frequency at $6 \mathrm{GHz}$.

\section{Antenna Design}

2.1. Design and Analysis of Antenna Element. At first, we have designed a traditional antipodal antenna and optimized it, but the gain and bandwidth cannot satisfy our goals. Thus, a novel antipodal Vivaldi antenna with tapering serrated structure instead of the classical antipodal antennas had been designed. The geometry of the antipodal Vivaldi antenna 


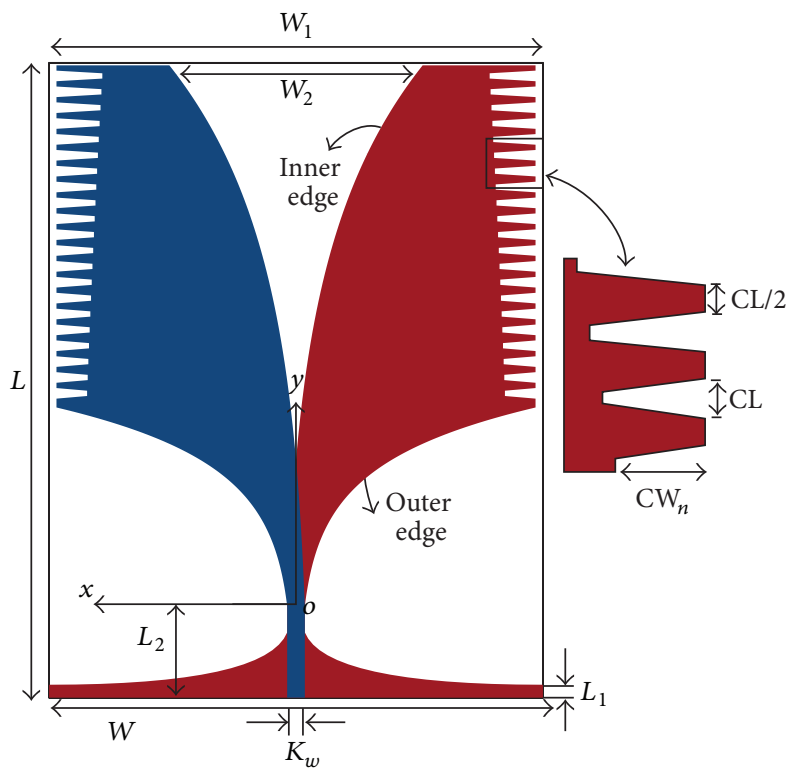

FIGURE 1: Geometry of the designed antenna element.

element is shown in Figure 1. In order to obtain a wider operating frequency range and lower reflection coefficient, a transition from a tapering microstrip line to the double parallel strip is used to match the impedance of the antenna. The end of the microstrip line is soldered with a SMA connector as a feeding port and connected to a coaxial cable.

Two symmetric logarithmic tapered patches lie on different substrate layers and the shape of these two patches is crucial for obtaining the required bandwidth [13], side lobes, and back lobes over the operating frequency range $[14,15]$, especially the value of $W_{2}$. The inner and outer edges of the taper are defined as

$$
\begin{array}{ll}
y_{i}=K_{i} \cdot \log \left(x_{i}+1+\frac{K_{w}}{2}\right) & x_{i}=-\frac{K_{w}}{2} \text { to } \frac{W_{2}}{2}, \\
y_{o}=K_{o} \cdot \log \left(x_{o}+1-\frac{K_{w}}{2}\right) & x_{o}=\frac{K_{w}}{2} \text { to } \frac{W_{1}}{2},
\end{array}
$$

where $\left(x_{i}, y_{i}\right)$ and $\left(x_{o}, y_{o}\right)$ are the coordinates of the inner and outer edges, respectively.

In this paper, a novel tapering periodic serrated structure is utilized at both the edges of the antipodal Vivaldi antenna to achieve a higher gain and wider bandwidth. The shapes of the slits are isosceles trapezoids, and the distance of adjacent slits is 1.5CL. Furthermore, the widths of the adjacent serrated structures are controlled by function of $\mathrm{CW}_{n}=s \cdot \mathrm{CW}_{n-1}$.

Table 1 shows the variables and dimensions of the designed antenna which are obtained through massive numerical calculations. As shown in Figure 2(a), although the reflection coefficient of the designed antenna is higher than that without serrated structure in high frequency, they are both below $-10 \mathrm{~dB}$ from $2.5 \mathrm{GHz}$ to $10.6 \mathrm{GHz}$ which contains the entire UWB spectrum (3.1-10.6 GHz). Furthermore, Figure 2(b) illustrates that the designed antenna can improve the gain from $2.5 \mathrm{GHz}$ to $8 \mathrm{GHz}$, especially from $4.5 \mathrm{GHz}$ to
TABLE 1: Variables and dimensions of the designed antenna.

\begin{tabular}{lcc}
\hline & $\begin{array}{c}\text { Without serrated } \\
\text { structure }\end{array}$ & $\begin{array}{c}\text { With tapering } \\
\text { serrated structure }\end{array}$ \\
\hline$L$ & $90 \mathrm{~mm}$ & $90 \mathrm{~mm}$ \\
$W$ & $70 \mathrm{~mm}$ & $70 \mathrm{~mm}$ \\
$L_{1}$ & $1.8 \mathrm{~mm}$ & $1.8 \mathrm{~mm}$ \\
$L_{2}$ & $11.2 \mathrm{~mm}$ & $11.2 \mathrm{~mm}$ \\
$W_{1}$ & $68 \mathrm{~mm}$ & $68 \mathrm{~mm}$ \\
$W_{2}$ & $34 \mathrm{~mm}$ & $34 \mathrm{~mm}$ \\
$K_{w}$ & $2.5 \mathrm{~mm}$ & $2.5 \mathrm{~mm}$ \\
$K_{o}$ & 8 & 8 \\
$K_{i}$ & 25.5 & 25.5 \\
$\mathrm{CL}$ & - & $1.5 \mathrm{~mm}$ \\
$\mathrm{CW}$ & - & $6.5 \mathrm{~mm}$ \\
$s$ & - & 0.98 \\
$n_{\max }$ & - & 21 \\
\hline
\end{tabular}

$5.5 \mathrm{GHz}$. The increment of gain reaches $1 \mathrm{dBi}$ to $2.6 \mathrm{dBi}$ at $4.5-$ $7.5 \mathrm{GHz}$.

The $E$ and $H$ plane radiation patterns at 5, 6, and $7 \mathrm{GHz}$ are shown in Figure 3, which proves that the designed antenna element has a good UWB feature. The $3 \mathrm{~dB}$ beam width of this antenna element on the $E$ plane is lower than $40^{\circ}$ at the operation frequency.

2.2. Design and Analysis of $2 \times 2$ CP Antenna Array. The calculated gain along the boresight of the designed Vivaldi antenna element from $4.5 \mathrm{GHz}$ to $7.5 \mathrm{GHz}$ varies from $9 \mathrm{dBi}$ to $11.5 \mathrm{dBi}$ in Figure 2(b). In fact, a common Vivaldi antenna is a pure linear polarization (LP) and directional antenna. In order to radiate $\mathrm{CP}$ wave in the maximum radiation direction 


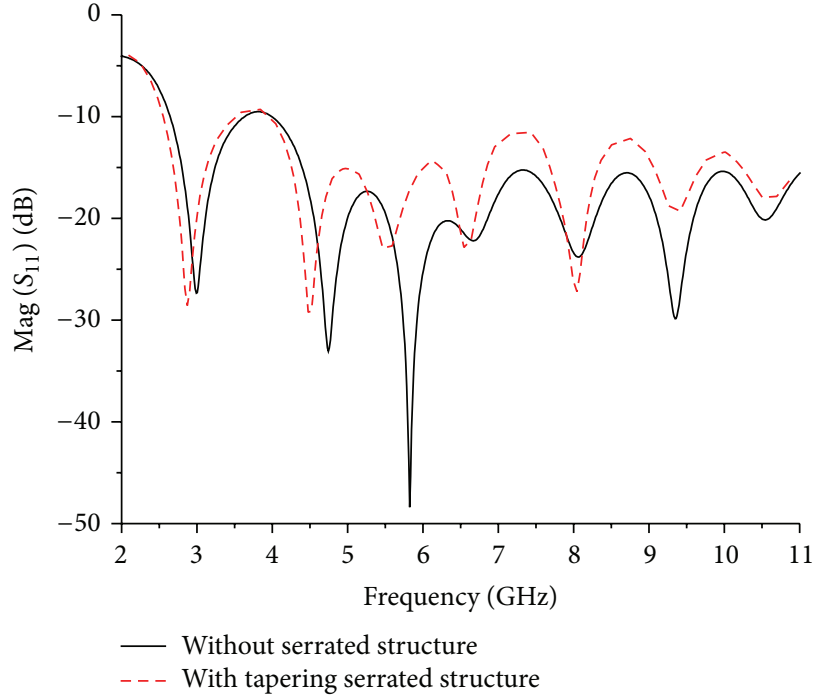

(a)

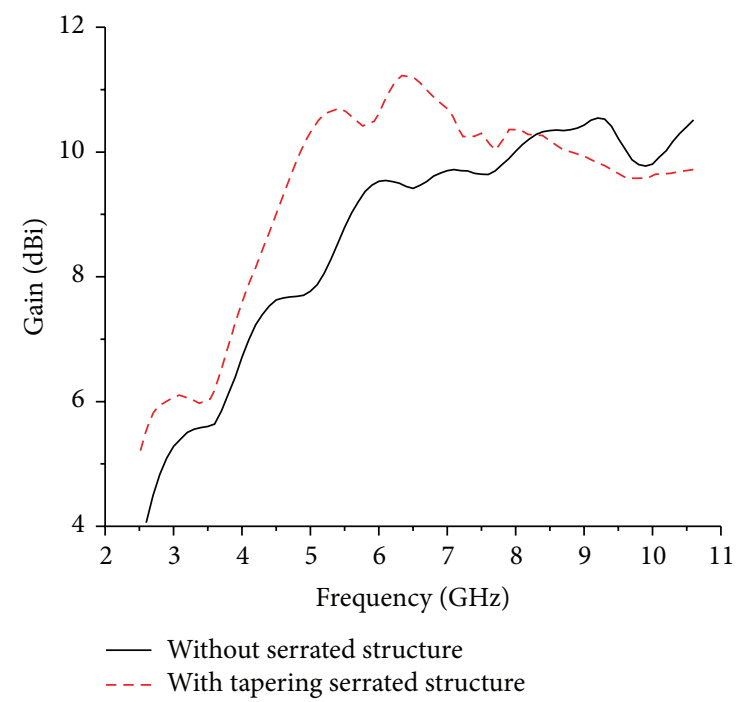

(b)

Figure 2: (a) Simulated reflection coefficient versus frequency for two structures. (b) Simulated gain versus frequency for two structures.
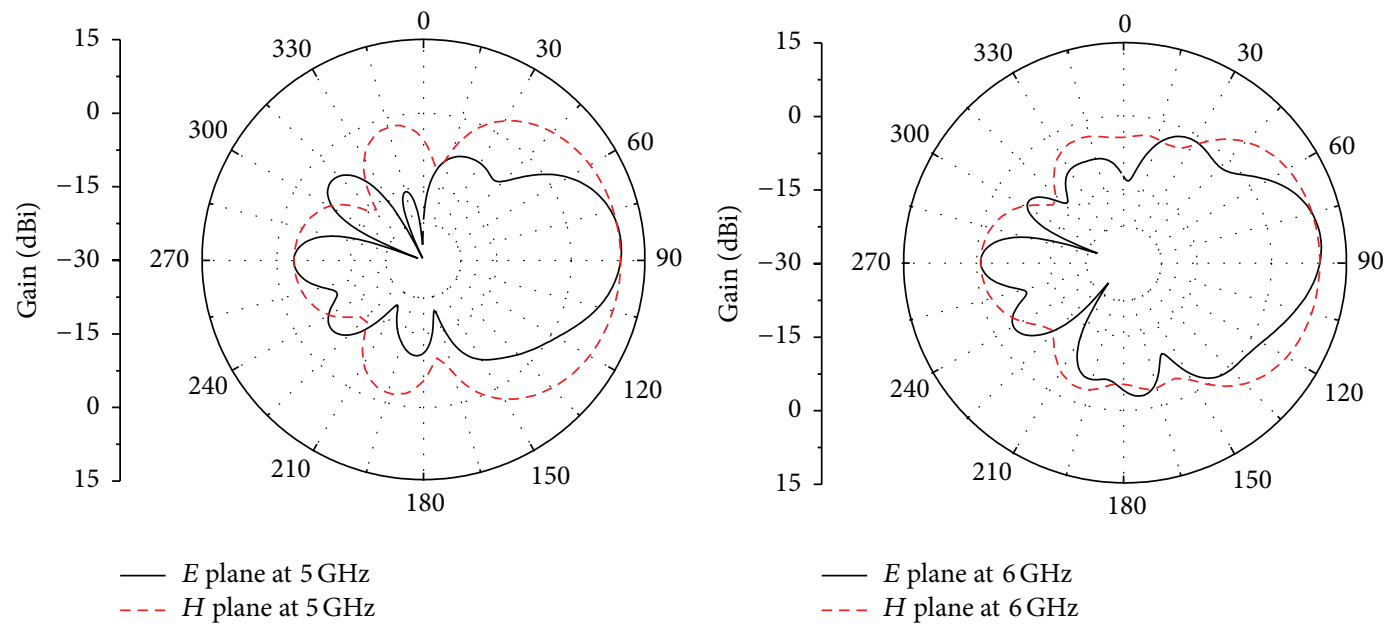

(a)

(b)

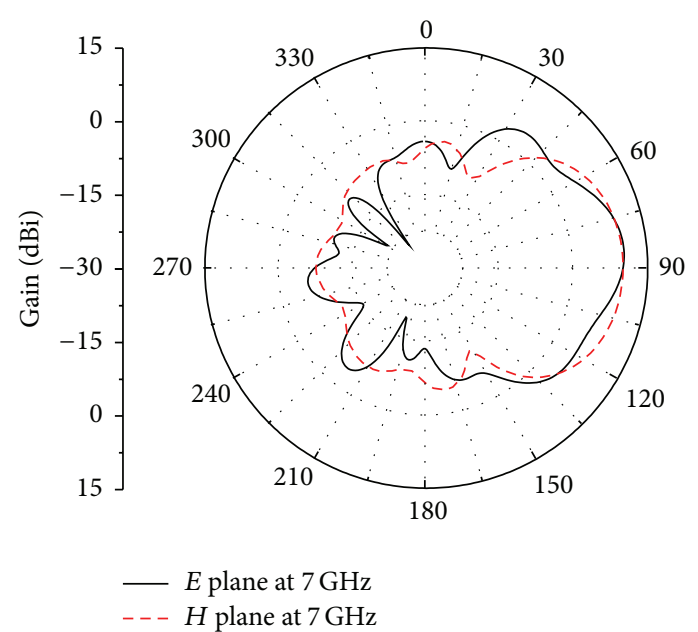

(c)

FIGURE 3: Simulated radiation patterns of antenna element on $E$ plane and $H$ plane at (a) $5 \mathrm{GHz}$, (b) $6 \mathrm{GHz}$, and (c) $7 \mathrm{GHz}$. 


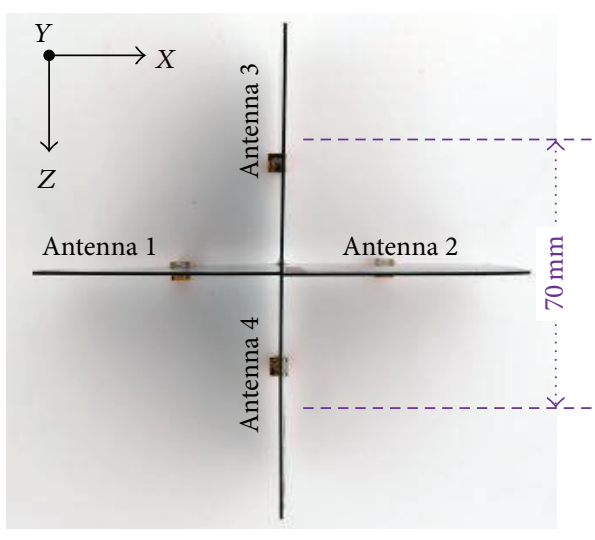

(a)

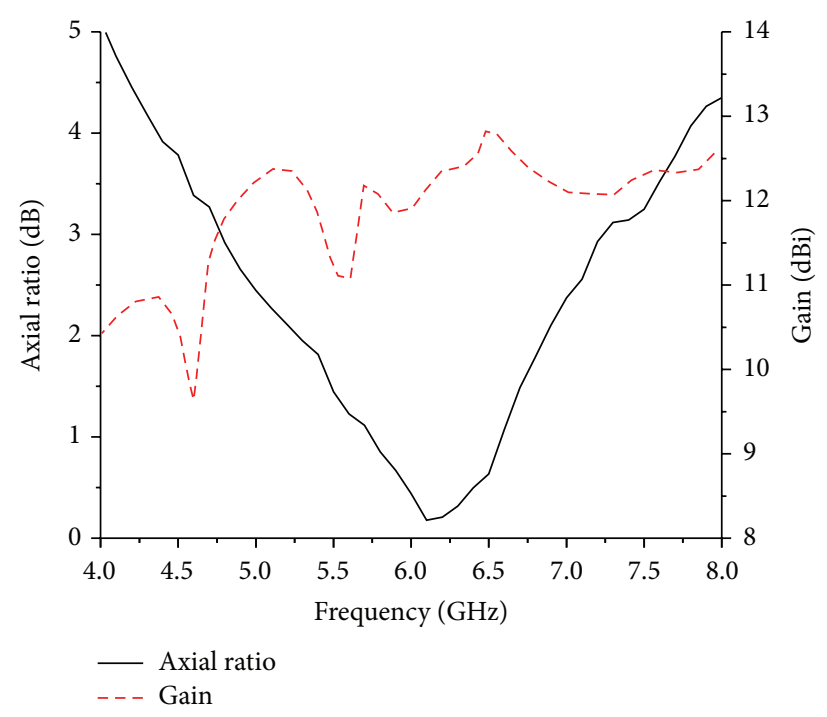

(b)

Figure 4: (a) Top view of the antenna array. (b) Simulated axial ratio and gain of the array.

and improve the antenna gain, a $2 \times 2$ antenna array with orthorhombic structure is promoted in this paper. As shown in Figure 4(a), the antenna array is composed of four identical Vivaldi antenna elements. If the antenna elements 1 and 2 have $90^{\circ}$ phase lag compared to antenna elements 3 and 4 , the array will excite left hand circular polarization (LHCP) in the maximum radiation direction. If there is $90^{\circ}$ phase lead instead of $90^{\circ}$ phase lag, the array will radiate the right hand circular polarization (RHCP) radiation wave. The gain of the array increases by $3 \mathrm{~dB}$ compared with the single antenna in theory.

The antenna array is simulated and optimized by using finite-difference time-domain method. The simulated axial ratio and gain of the antenna array are shown in Figure 4(b). The simulated $3 \mathrm{~dB}$ axial ratio bandwidth is as high as $2.5 \mathrm{GHz}$, which is about $42.0 \%$ with respect to the center frequency at $6 \mathrm{GHz}$. The gain of the antenna array increases by about $3 \mathrm{~dB}$ compared with the Vivaldi antenna element.

Figure 5 shows the simulated radiation patterns of the antenna array operating at 5, 6, and $7 \mathrm{GHz}$, respectively. Since we feed the antenna array elements with equal amplitude and $90^{\circ}$ phase lag for antenna elements 1 and 2 compared with antenna elements 3 and 4, a LHCP characteristic is obtained in the maximum radiation direction. This LHCP antenna has a lower side lobe level and a fine front-to-back ratio within its operating frequency range. Furthermore, the radiation level under a RHCP condition is much lower compared to that under the LHCP condition in the maximum radiation direction, which also verifies the validity of the designed method.

\section{Fabrication and Measurements}

An F4B substrate has been chosen since its dielectric constant is stable and dielectric loss is low over a broad frequency range and the cost is low. As shown in Figure 6, the designed Vivaldi antenna and CP antenna array are realized on the substrates with dielectric constant of 2.65 and thickness of $1 \mathrm{~mm}$. The Vivaldi antenna element is compact with a dimension of $89 \mathrm{~mm}$ by $68 \mathrm{~mm}$.

An Agilent N5230A vector network analyzer is used to measure the $S$-parameters of these antennas. As shown in Figure 7, the measured reflection coefficient of the designed Vivaldi antenna element is lower than $-10 \mathrm{~dB}$ from $2.3 \mathrm{GHz}$ to $11 \mathrm{GHz}$, and the measured $-10 \mathrm{~dB}$ impedance bandwidth of the $\mathrm{CP}$ antenna array fully covers the desired band from $4.5 \mathrm{GHz}$ to $7.5 \mathrm{GHz}$.

Radiation patterns at 5,6 , and $7 \mathrm{GHz}$ are measured to verify the ultrawide band characteristic of Vivaldi antenna element. Furthermore, comparisons of the data on the coplane and cross-plane, as shown in Figure 8, also prove the good performance of LP characteristic.

Figure 9 shows normalized measured and simulated radiation patterns of the antenna array at operation frequencies. A standard LP antenna is utilized as the transmitting antenna of the measurement setup. Simulated and measured results agree well which also indicates the $\mathrm{CP}$ characteristic of the designed antenna array.

The simulated and measured gains of the LP Vivaldi antenna element are shown in Figure 10(a). The measured gains of the LP Vivaldi antenna array has a smaller increase than the gain of simulation since there are couplings between the two units in the parallel direction. A maximum measured gain of $10.5 \mathrm{dBi}$ is obtained at $6.5 \mathrm{GHz}$. Figure $10(\mathrm{~b})$ shows the simulated and measured axial ratio of the $\mathrm{CP}$ antenna array. The radiation antennas of two orthogonal directions have a $90^{\circ}$ phase difference. The bandwidth with phase difference of $90^{\circ}$ between the ports of the Wilkinson power divider determines the axial ratio parameters of the circular polarization array. The measured $3 \mathrm{~dB}$ axial ratio bandwidth 

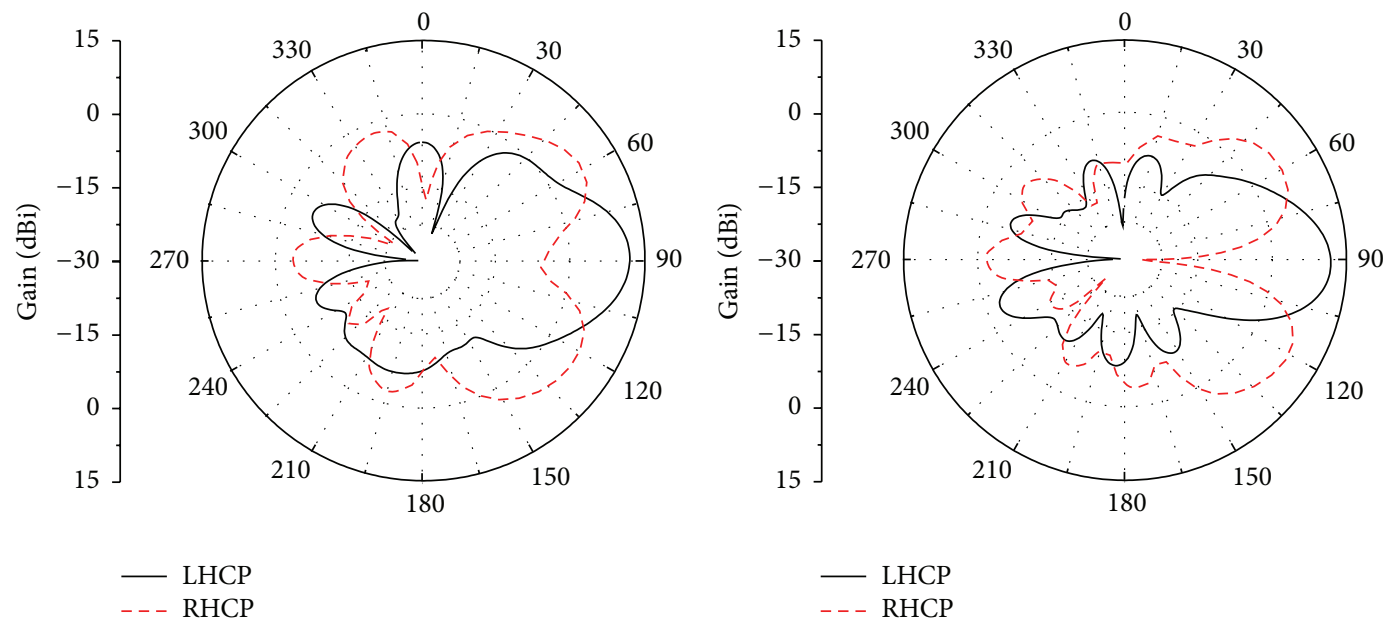

(a)

(b)

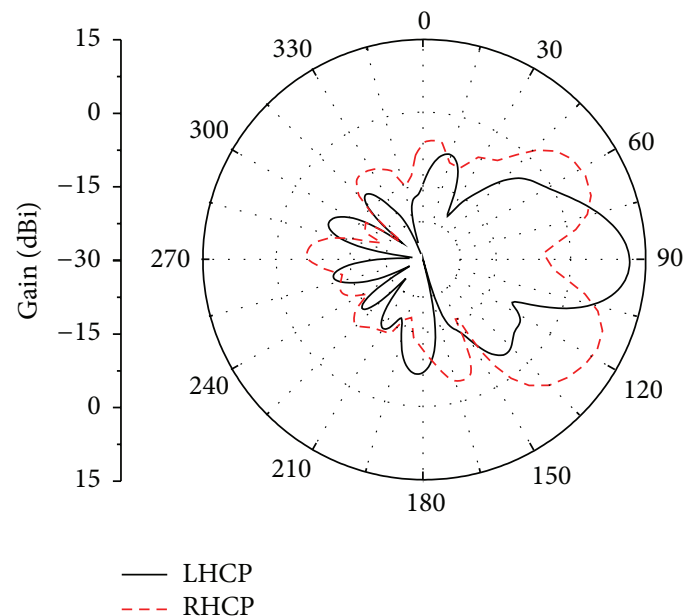

(c)

FIGURE 5: Simulated radiation patterns of antenna array at (a) $5 \mathrm{GHz}$, (b) $6 \mathrm{GHz}$, and (c) $7 \mathrm{GHz}$.
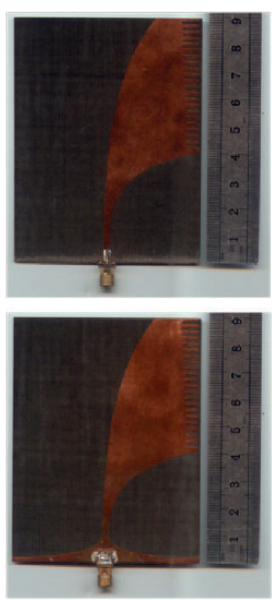

(a)

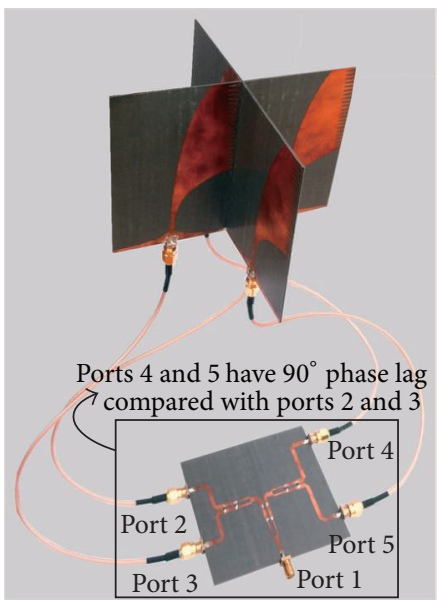

(b)

Figure 6: (a) Top and bottom views of the single antenna. (b) The photograph of the antenna array. 


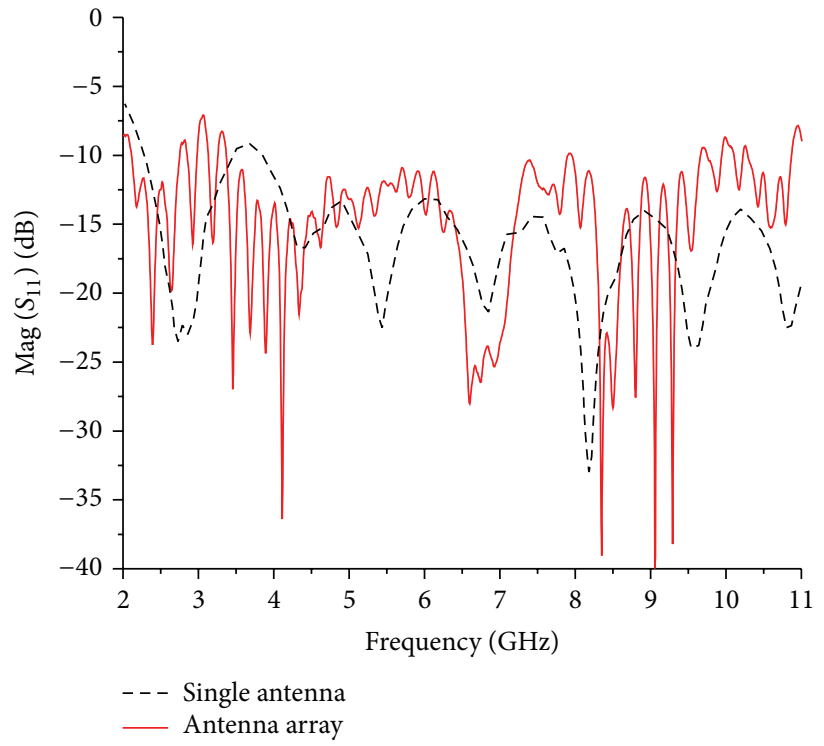

Figure 7: Measured reflection coefficient of single antenna and antenna array.

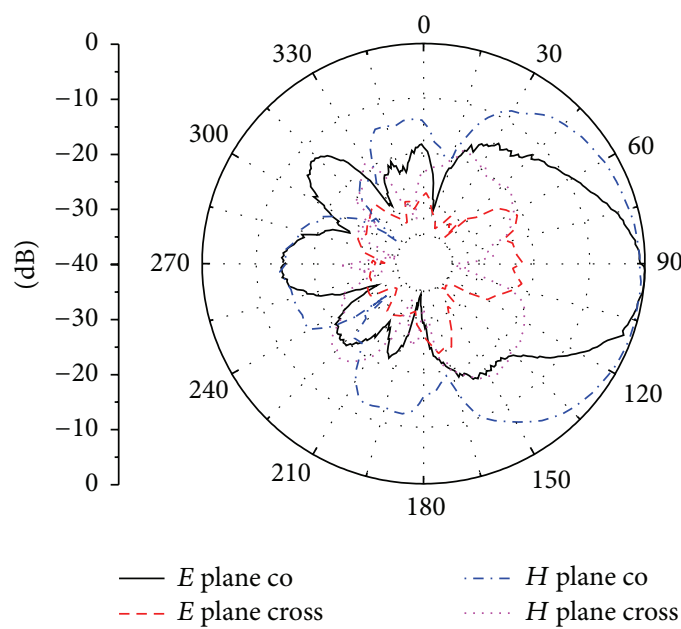

(a)

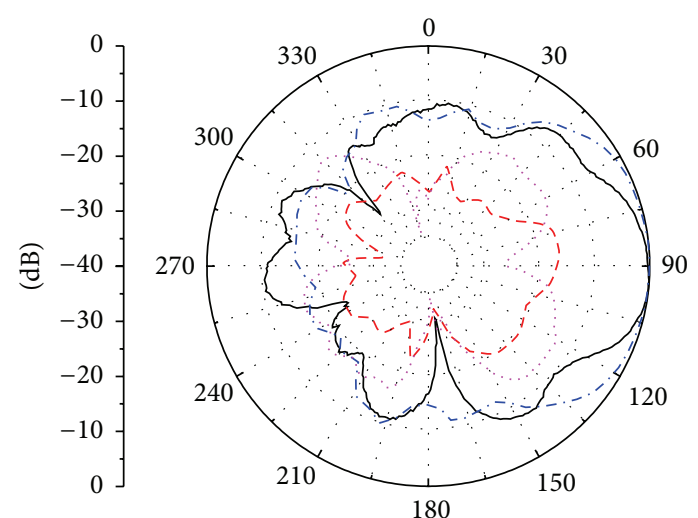

- E plane co

- - E plane cross

(b)

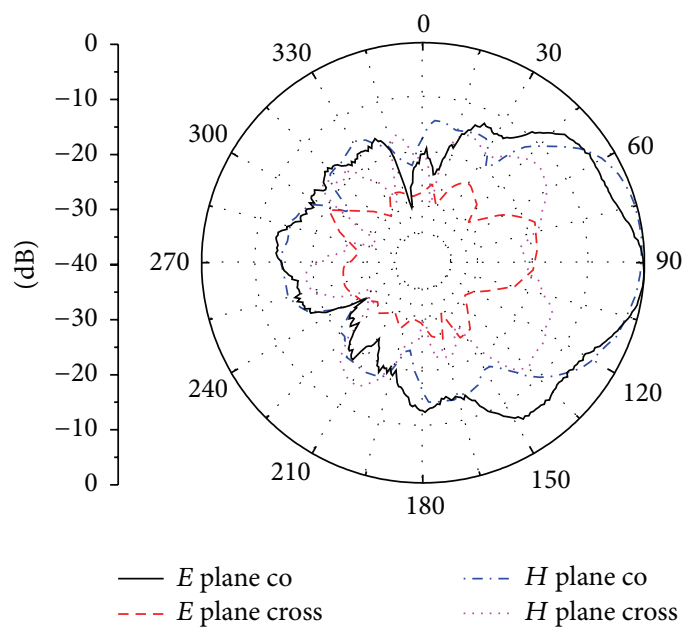

(c)

FIGURE 8: Normalized measured radiation patterns of single antenna at (a) $5 \mathrm{GHz}$, (b) $6 \mathrm{GHz}$, and (c) $7 \mathrm{GHz}$. 


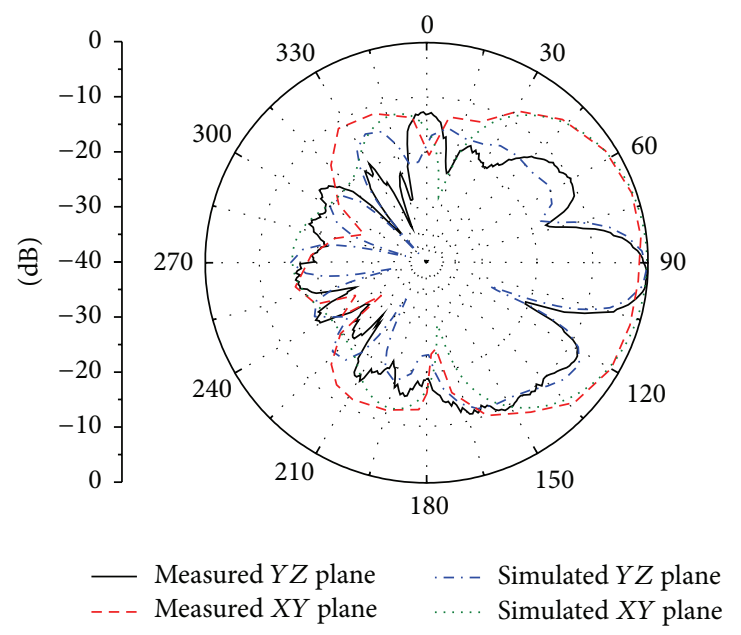

(a)
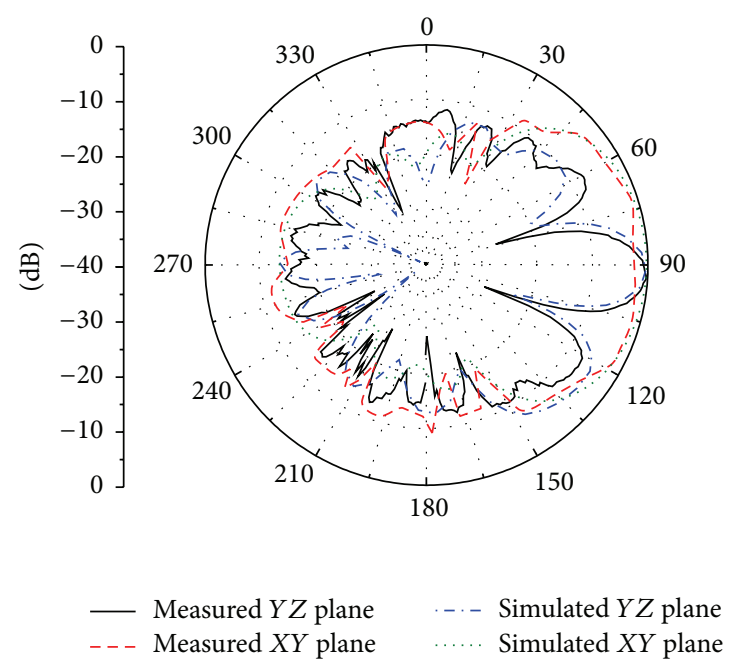

(b)

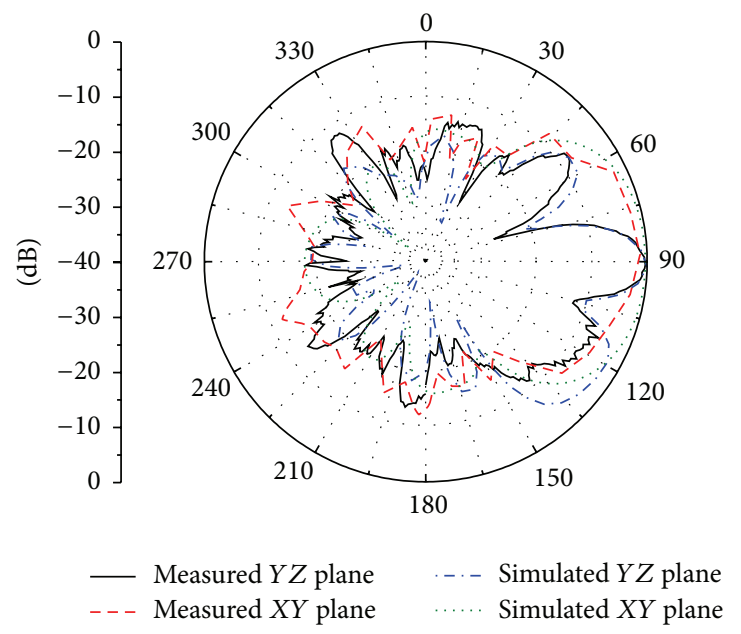

(c)

FIGURE 9: Normalized measured and simulated radiation patterns of array at (a) $5 \mathrm{GHz}$, (b) $6 \mathrm{GHz}$, and (c) $7 \mathrm{GHz}$.

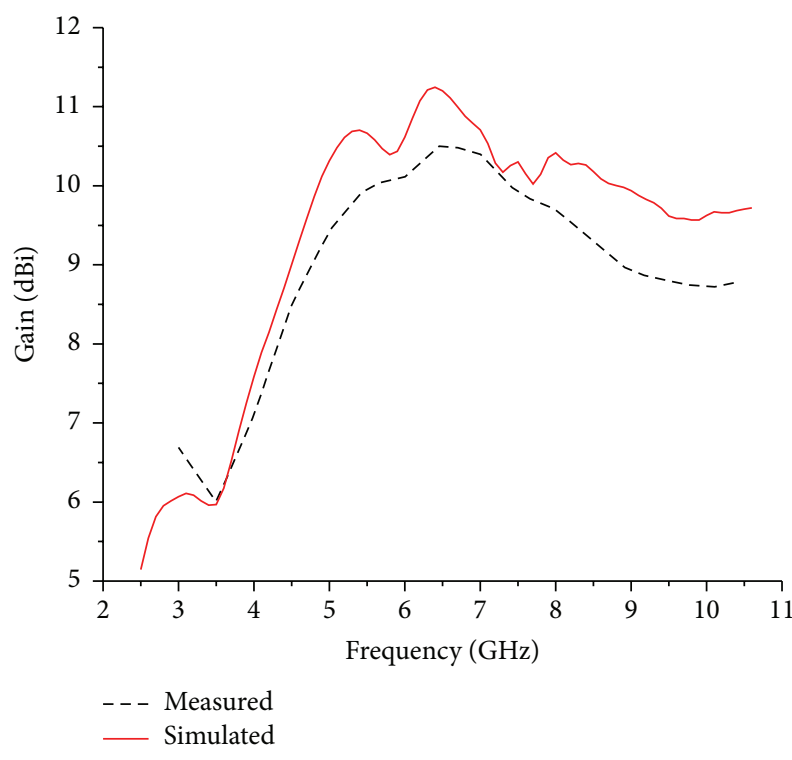

(a)

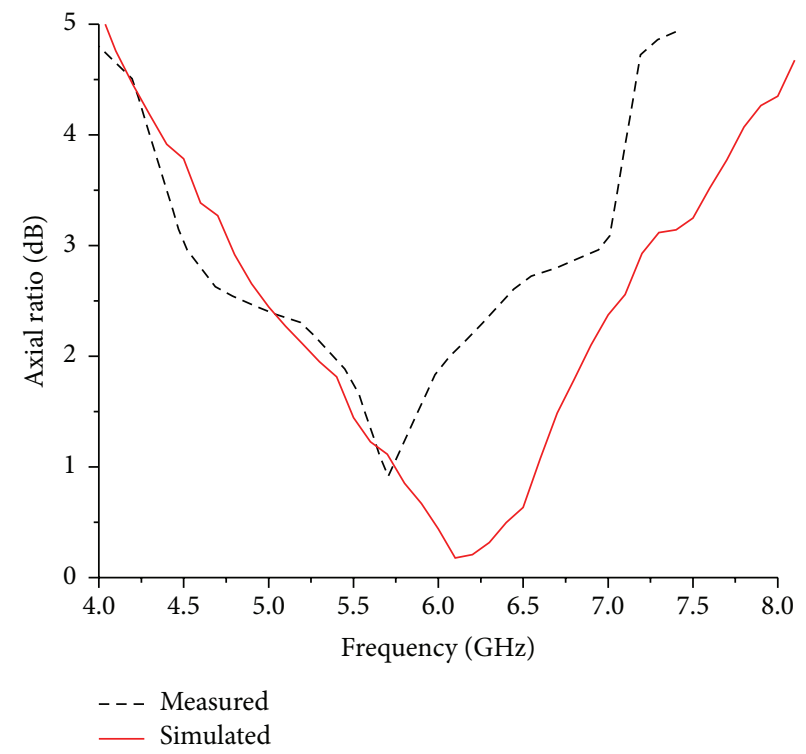

(b)

FIGURE 10: (a) The gain of the LP Vivaldi antenna element and (b) the axial ratio of the CP antenna array. 
reaches about $42 \%$ from $4.5 \mathrm{GHz}$ to $7.0 \mathrm{GHz}$. The phase difference was generated by the microstrip power divider. The power divider and antenna are connected through coaxial cables. In theory, the four coaxial cables own the same phase characteristics. In practice, the phase difference of the connection structure will affect the characteristics of the feed which leads to a frequency shifting. Thus, there is a frequency shifting shown in Figure 10(b).

\section{Conclusion}

A novel high-gain UWB antipodal Vivaldi antenna with a tapering serrated structure is presented. Compared with the traditional Vivaldi antenna without serrated structure, the designed antenna shows an obvious promotion of the gain in the desired frequency band $(4.5-7.5 \mathrm{GHz})$. Furthermore, associating with a $90^{\circ}$ phase-shifting $6 \mathrm{~dB}$ Wilkinson power divider, a $2 \times 2$ antenna array constructed with four Vivaldi antennas is designed and fabricated to achieve a CP characteristic. The $3 \mathrm{~dB}$ axial ratio bandwidth of this $\mathrm{CP}$ antenna array reaches about $42 \%$ with respect to the center frequency at $6 \mathrm{GHz}$. It will be applied in the field of commercial or military communication, remote sensing, and so forth.

\section{Conflict of Interests}

The authors declare that there is no conflict of interests regarding the publication of this paper.

\section{Acknowledgments}

This work was supported in part by the 973 Program 2013CB328902, NSFC 61271074, and NCET-12-0383.

\section{References}

[1] K. Kiminami, A. Hirata, and T. Shiozawa, "Double-sided printed bow-tie antenna for UWB communications," IEEE Antennas and Wireless Propagation Letters, vol. 3, no. 1, pp. 152153, 2004.

[2] K.-L. Wong, C.-H. Wu, and S.-W. Su, "Ultrawide-band square planar metal-plate monopole antenna with a trident-shaped feeding strip," IEEE Transactions on Antennas and Propagation, vol. 53, no. 4, pp. 1262-1269, 2005.

[3] P. J. Gibson, "The vivaldi aerial," in Proceedings of the 9th European Microwave Conference, pp. 101-105, Brighton, UK, September 1979.

[4] A. Z. Hood, T. Karacolak, and E. Topsakal, "A small antipodal vivaldi antenna for ultrawide-band applications," IEEE Antennas and Wireless Propagation Letters, vol. 7, pp. 656-660, 2008.

[5] J. B. Rizk and G. M. Rebeiz, "Millimeter-wave Fermi tapered slot antennas on micromachined silicon substrates," IEEE Transactions on Antennas and Propagation, vol. 50, no. 3, pp. 379-383, 2002.

[6] Z. Wang and H. Zhang, "Improvements in a high gain UWB antenna with corrugated edges," Progress in Electromagnetics Research C, vol. 6, pp. 159-166, 2009.

[7] Z. Briqech and A. Sebak, " $60 \mathrm{GHz}$ Fermi tapered slot antenna with sin-corrugation," in Electrical and Computer Engineering, H3G 1M8, Concordia University, Montreal, Canada, 2013.
[8] G. Zhang, H. Zhang, Z. Wang, and Z. Yuan, "Improvements in a 4-element high gain directional UWB antenna array," Journal of Electromagnetic Waves and Applications, vol. 24, no. 4, pp. 453$461,2010$.

[9] S. Sugawara, Y. Maita, K. Adachi, K. Mori, and K. Mizuno, "Characteristics of a mm-wave tapered slot antenna with corrugated edges," in Proceedings of the IEEE MTT-S International Microwaves Symposium Digest, vol. 2, pp. 533-536, June 1998.

[10] M. Cui, Y. Wu, Z. Zhang et al., "A Ka band circularly polarized corrugated antipodal tapered slot antenna," in Proceedings of the 3rd Asia-Pacific Conference on Antennas and Propagation, pp. 294-297, Harbin, China, July 2014.

[11] P. Fei, Y.-C. Jiao, W. Hu, and F.-S. Zhang, "A miniaturized antipodal vivaldi antenna with improved radiation characteristics," IEEE Antennas and Wireless Propagation Letters, vol. 10, pp. 127-130, 2011.

[12] Y. Wu, J. Lu, Y. Liu, and H. Yang, "Modified design of the antipodal Vivaldi antenna," in Proceedings of the 10th International Symposium on Antennas, Propagation \& EM Theory (ISAPE '12), pp. 316-319, IEEE, Xi'an, China, October 2012.

[13] J. Bai, S. Shi, and D. W. Prather, "Modified compact antipodal Vivaldi antenna for 4-50-GHz UWB application," IEEE Transactions on Microwave Theory and Techniques, vol. 59, no. 4, pp. 1051-1057, 2011.

[14] Y. Takagi, H. Sato, Y. Wagatsuma, K. Sawaya, and K. Mizuno, "Study of high gain and broadband antipodal Fermi antenna with corrugation," in Proceedings of the International Symposium on Antennas and Propagation, vol. 1, pp. 69-72, Sendai, Japan, 2004.

[15] R. Herzi, R. Gharbi, H. Zairi, and A. Gharsallah, "A tuneable Antipodal Vivaldi antenna for UWB application," in Proceedings of the 10th International Multi-Conference on Systems, Signals \& Devices (SSD '13), pp. 1-4, Hammamet, Tunisia, March 2013. 

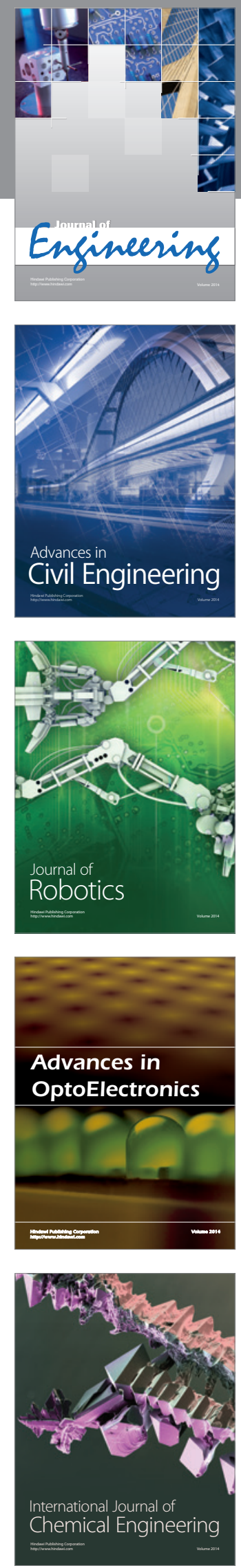

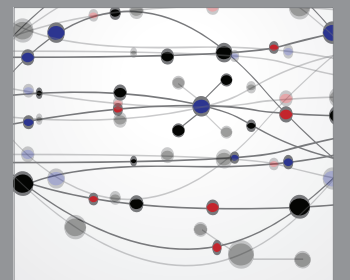

The Scientific World Journal
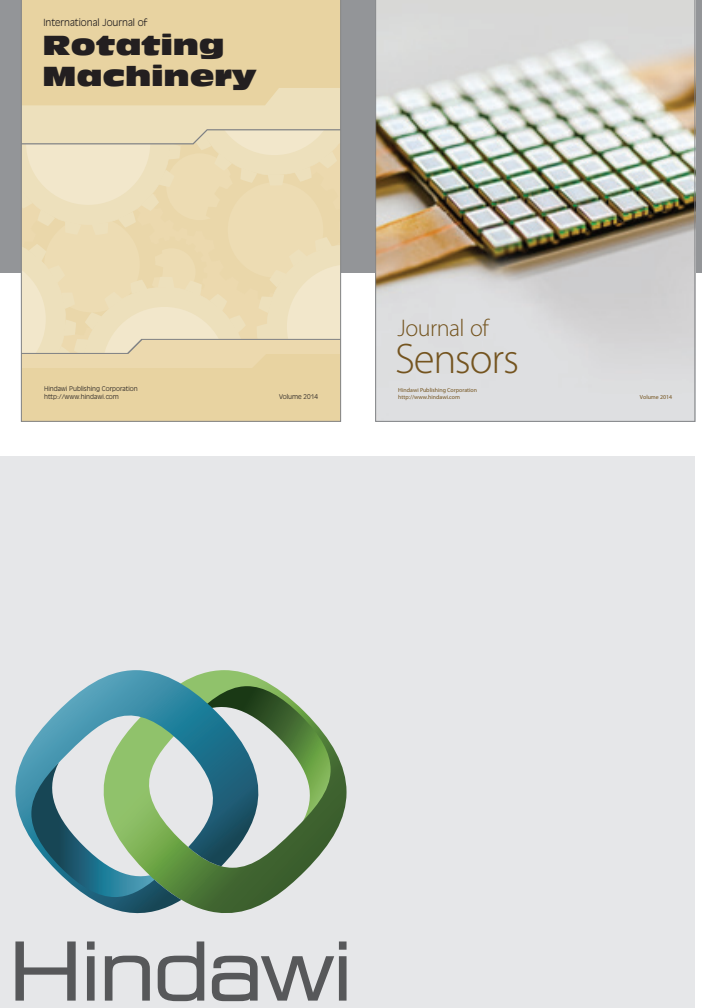

Submit your manuscripts at http://www.hindawi.com
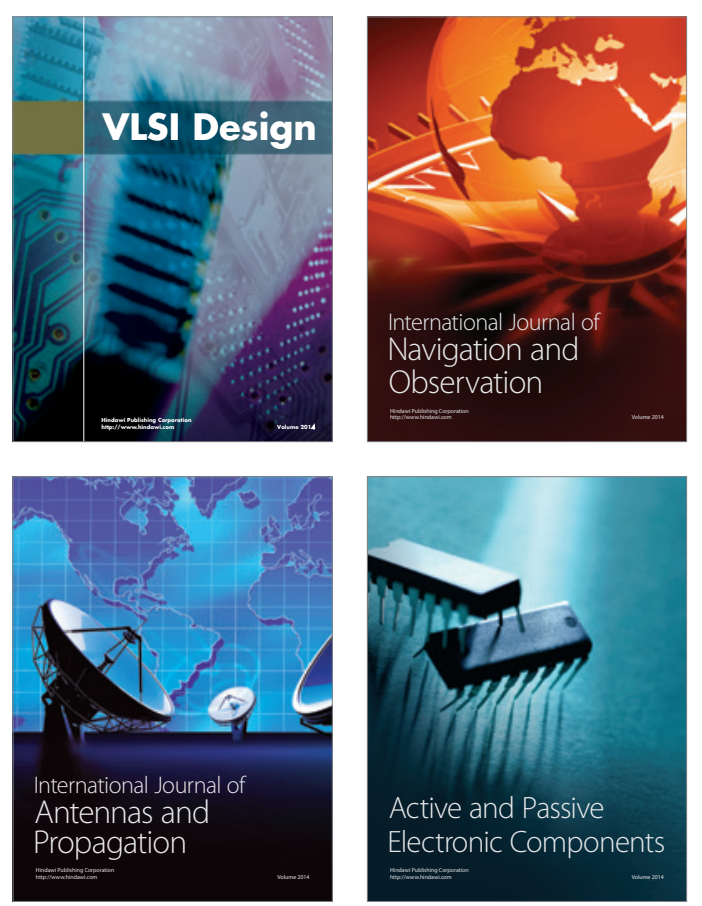
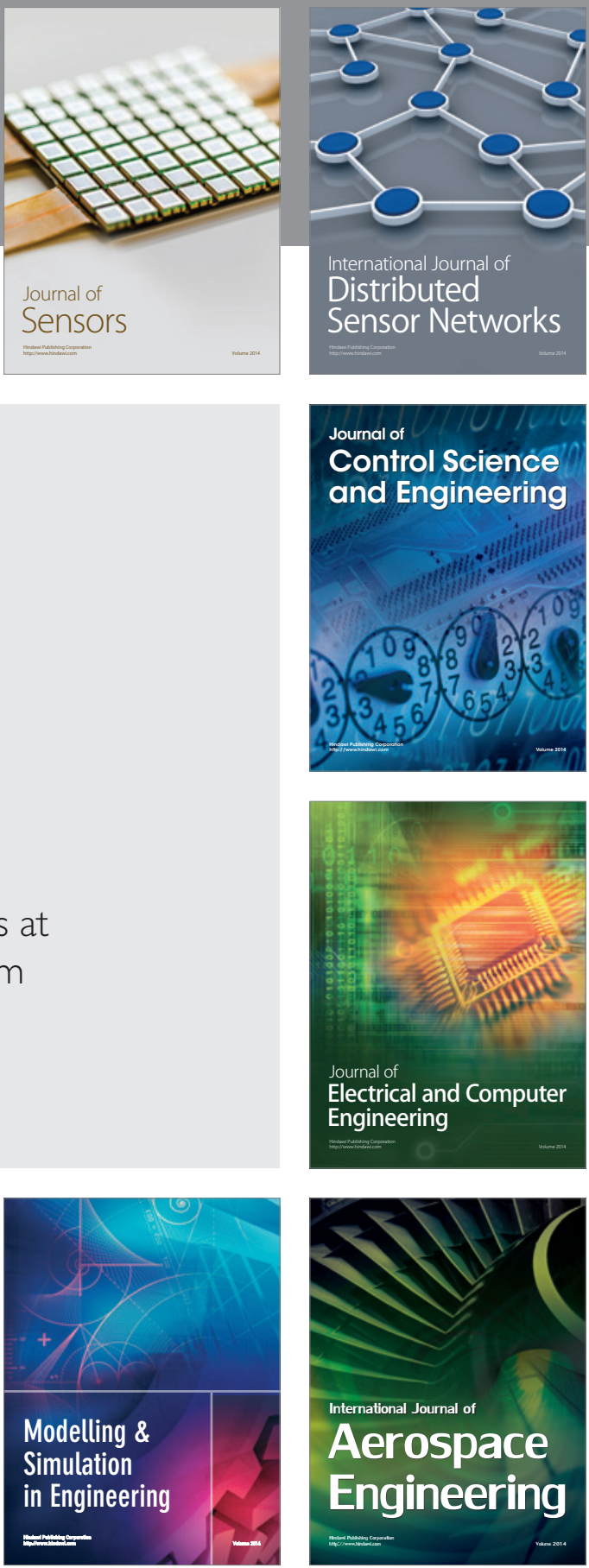

Journal of

Control Science

and Engineering
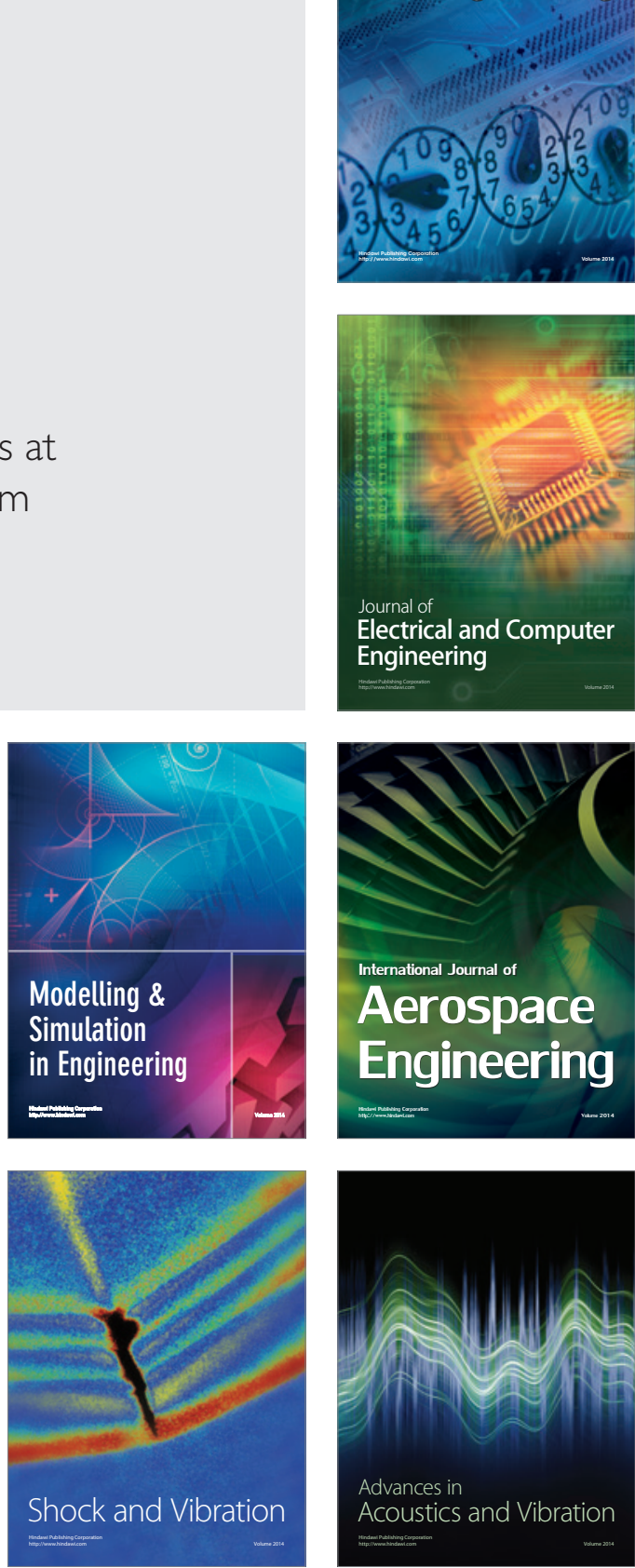